\title{
Seroprevalence of SARS-COV-2 Antibodies in Medical Staff, South-West of Iran
}

\author{
Amir Emami ${ }^{1 *}{ }^{\circledR}$, Fatemeh Javanmardi $^{\circledR}$, Neda Pirbonyeh $^{1}{ }^{\circledR}$, Sedigheh Moradi Ghermezi $^{1}$, Tahereh Rezaei ${ }^{2}$, \\ Hamid Bakhtiari ${ }^{3}$, Ali Mansouri ${ }^{2}$
}

${ }^{1}$ Microbiology Department, Burn and Wound Healing Research Center, Shiraz University of Medical Sciences, Shiraz, Iran ${ }^{2}$ Vice Chancellor for Treatment, Shiraz University of Medical Sciences, Shiraz, Iran

${ }^{3}$ Vice Chancellor for Health Affairs Center of Disease Control (CDC), Shiraz University of Medical Sciences, Shiraz, Iran

\section{*Correspondence to Amir Emami, Email: emami. microbia@gmail.com}

Received August 18, 2021 Accepted September 11, 2021 Published online September 30, 2021

\begin{abstract}
Introduction: Serological assay has critical role in defining immunity based on seroprevalence. Identifying the extend of seropositivity would determine the truly infected cases.

Method: We conducted serologic testing for SARS-COV-2 antibodies in 492 health care workers (HCWs) before vaccination program in Shiraz, Iran (30 April 2020).

Results: Based on job position, cases were divided into two categories: frontline staff (169, $34.34 \%)$ and non-front-line staff $(323,65.65 \%)$. Of all cases 40.65 were female and the rest of them were male. Mean age was estimated $47.97 \pm 109.20$ and $38.98 \pm 8.76$ in high risk and low risk population respectively $(P=0.1)$. In total $367(74.59 \%)$ subjects reported to suffered at least one of the COVID-19 symptoms. More than half of cases (73.57) had history of COVID-19 disease within six months before this survey. No relation was seen between blood type and immunoglobulin positivity. Seropositivity prevalence in low risk and high-risk populations were estimated 8.0 (95\% Cl: 1.0-16.8) and 5.2(95\% Cl: 1.2-9.8) respectively. The immunoglobulin test for SARS-COV-2 revealed that $64(64 / 492,13 \%)$ and $37(37 / 492,7.52 \%)$ subjects had $\operatorname{lgG}$ and IgM antibodies against the virus. $14(14 / 169,8.28 \%)$ individuals who had IgM were frontline health workers, this is while $23(14 / 323,7.12 \%)$ were non front line

Conclusion: The findings of this study imply that seropositivity was lower in non-frontline health care staff. Furthermore, there was no association between blood type and seropositivity. Therefore, the knowledge of antibody status may help into overcome fears in clinical staff, and also more attention is need for interpreting the levels of anti-SARS-CoV-2 antibodies to make a clinical diagnosis of COVID-19, since the time of expose and time of test is an important point which should be considered in the issue.

Keywords: COVID-19, SARS-COV-2, Antibodies, Serology
\end{abstract}

\section{Introduction}

It is more than 1 year that all over the world expose to a pandemic. The etiological agent of the current outbreak was a new Beta corona virus strain from the Coronaviridae family. COVID-19 remains as a major health concern threating all over the globe. ${ }^{1}$ One of the most striking aspect of COVID-19 is manifestation of disease, which vary in a wide range from asymptomatic, sometime apparently healthy, mild, moderate, sever and rapidly fatal. Therefore, it is important to detect carriers and mild cases, since this may be effective preventive measures. Immune response to the infection and knowing the host defense against SARS-COV-2 may help in understanding the pathophysiology of the disease and so better treatments. ${ }^{2}$

Hence, there is no exact information about proportion of asymptomatic case, the rate of transmission of SARS-COV-2 and so the causes of COVID-19, remain unclear yet. According to the World Health Organization (WHO); identifying the extend of seropositivity would determine the truly infected cases. ${ }^{3,4}$

Serological assay has critical role in definingimmunitybased on seroprevalence. However, it is unclear whether antibody response is significantly different among asymptomatic, mild, moderate or severe

(C) 2021 The Author(s); Published by Zabol University of Medical Sciences. This is an open-access article distributed under the terms of the Creative Commons Attribution License (http://creativecommons.org/licenses/by/4.0), which permits unrestricted use, distribution, and reproduction in any medium, provided the original work is properly cited. 
cases. ${ }^{5}$ Serological testing and screening especially for those who have close contact with COVID-19 cases will be useful in identifying the local transmission health care workers (HCWs) who are in frontline against COVID-19 pandemic, deemed to be at higher risk. Therefore, it is critical to monitor and screen their sero-immunity. ${ }^{6}$ Immunoglobulins have a critical role in the immune system. Based on the serological test, it is possible to detect the presence of antibodies in serum during days to weeks following acute infection. There are different ways to determine different aspect of immune response and functionality of antibodies. Immunoglobulin $M$ (IgM) antibodies are body's first response and produced when body's immune system detect a new infection. IgM start to increase and create a short term protection and then decrease as IgG begins to produce. IgG is the most common antibody in the blood (70\% -80\%). IgG have an important role for host defense system and humoral immunity. ${ }^{7}$ Assessing serology of COVID-19 will guide for making health policies to control the spreading of infection. This study is an initial phase of an assay which aims to evaluate the sero-immunity of HCWs in referral COVID-19 hospitals affiliated with Shiraz University of Medical Sciences before the vaccination program.

\section{Materials and Methods}

In this population based-study, we conducted serologic testing for SARS-COV-2 antibodies in HCWs before vaccination program in Shiraz, Iran (30 April 2020).

The sample size for this study was assessed based on the WHO sero-epidemiological investigation protocol. ${ }^{3}$ By considering seroprevalence rate of $13 \%$, design effect of 1.85 , total sample size was 492 participants as the sufficient sample size for this study. Foe each case, the presence of SARS-COV-2 specific immunoglobulin G and $\mathrm{M}$ antibodies were assessed in serum samples. Each participant completed the questionnaires and then $5 \mathrm{~mL}$ of capillary blood was taken for laboratory technique. Each tube was labeled with a unique participant ID and centrifuged immediately. For each participant two serums were stored at -80 , one or detecting $\operatorname{IgG}$ and the other for IgM. Pishtaz Teb SARS-COV-2 kits were used for conducting the tests, following the manufactory's instructions to evaluate SARS-CoV-2-specific immunoglobulin $\mathrm{G}$ and $\mathrm{M}$. The test principle is based on indirect ELISA (enzyme-linked immunosorbent assay) technique. In this technique, microwells are coated with a certain amount of $\mathrm{N}$ (nucleocapsid) antigen of the SARSCoV-2 virus. Then the diluted serum sample is allowed to react with solid-phase antigens. If specific antibodies are present in the sera, they will bind to the SARS-CoV-2 $\mathrm{N}$ antigens. The assay is to be considered valid if three following criteria met: First, the OD $(450 \mathrm{~nm})$ of less than 0.05 for blank. Higher values indicate chromogen substrate contamination. Second, the OD $(450 \mathrm{~nm})$ of less than 0.1 for negative control serum. Higher values indicate poor operation of the washing step. If so, repeat the procedure with more attention to the washing step. Third, the OD $(450 \mathrm{~nm})$ of more than 0.6 for positive control serum. Then, Calculate the cut off values using the following formula:

Cut-off value $=$ Negative control mean $O D+0.15$

To determine positive or negative results, calculate Cut off index or each sample using the following formula: Cut-off Index $(C O I)=O D$ of sample / Cut-off value

The sensitivity and specificity for SARS-CoV-2 IgG or IGM ELISA kit was considered $94.1 \%$ and $98.3 \%$ respectively.

\section{Statistical Analysis}

Descriptive statistics for baseline characteristics were reported by frequency (\%) and Mean and standard deviation. In order to assess the seroprevalence of SARSCoV-2 antibodies, the population was divided into two categories: first, high risk population who is in front line and has close contact with positive COVID-19 patients consist of nursed and physicians. Second, low risk population who are randomly selected from hospital staff consist of cashier, pharmacy staff. Chi-square test was used for evaluating the proportion between two groups. Independent T-test was used for comparison quantity variables. Furthermore, seroprevalence and its 95\% confidence interval were estimated by use of exact binomial models. All statistical analysis was done by STATA 13.

\section{Results}

In total, 492 individuals participated in this study. Based on job position cases were divided into two categories: frontline staff $(169,34.34 \%)$ and non-front-line staff (323, $65.65 \%)$. Of all cases 40.65 were female and the rest of them were male. Mean age was estimated $47.97 \pm 109.20$ and $38.98 \pm 8.76$ in high risk and low risk population respectively. In total 367 (74.59\%) subjects reported to suffered at least one of the COVID-19 symptoms. Most recent symptoms which was significantly different between two mentioned group including cough, chills, headache. shortness of breath and weakness. Moreover, (362/492, 73.57\%) cases had history of COVID-19 disease within six months before this survey. More details are provided in Table 1.

The immunoglobulin test for SARS-COV-2 revealed that $64(64 / 492,13 \%)$ and $37(37 / 492,7.52 \%)$ subjects had IgG and IgM antibodies against the virus. 14 (14/169, $8.28 \%$ ) individuals who had IgM were frontline health workers, this is while $23(14 / 323,7.12 \%)$ were non front line. More details are provided in Table 2.

Of all IgM seropositive cases, 19 (51.35\%) and 18 (48.64\%) were males and females respectively. 19 (51.35\%) cases had history of COVID-19 disease and 24 (64.86\%) cases had disease symptoms during the last six months. Of all 64 cases with IgG positive test, 27 (42.18\%) 
Table 1. Baseline Characteristics of Health Care Workers

\begin{tabular}{|c|c|c|c|}
\hline & $\begin{array}{l}\text { Front-Line Physicians and Nurses } \\
\qquad(\mathrm{n}=169)\end{array}$ & $\begin{array}{l}\text { Non-front-Line Health Care Workers } \\
\qquad(\mathrm{n}=323)\end{array}$ & $P$ Value \\
\hline Age & $47.97 \pm 109.20$ & $38.98 \pm 8.76$ & 0.1 \\
\hline \multicolumn{4}{|l|}{ Gender } \\
\hline Male & $121(71.6 \%)$ & $171(52.9 \%)$ & \multirow{2}{*}{0.001} \\
\hline Female & $48(28.4 \%)$ & $152(47.1 \%)$ & \\
\hline Height & $158.09 \pm 36.44$ & $163.42 \pm 30.84$ & 0.5 \\
\hline Weight & $63.41 \pm 24.05$ & $65.59 \pm 25.45$ & 0.1 \\
\hline Work shifts, N/wk & $4.76 \pm 2.29$ & $5.45 \pm 1.24$ & $<0.001$ \\
\hline \multicolumn{4}{|l|}{ Underlying disease } \\
\hline Cardiovascular disease (yes) & $6(3.55 \%)$ & $13(4.02 \%)$ & 0.8 \\
\hline Hypertension (yes) & $12(7.10 \%)$ & $28(8.66 \%)$ & 0.58 \\
\hline Lung Disease (yes) & $4(2.36 \%)$ & $12(3.71 \%)$ & 0.44 \\
\hline Asthma (yes) & $10(5.91 \%)$ & $14(4.33 \%)$ & 0.41 \\
\hline Diabetes (yes) & $7(4.14 \%)$ & $13(4.02 \%)$ & 0.9 \\
\hline Obesity (yes) & $26(15.38 \%)$ & $52(16.09 \%)$ & 0.9 \\
\hline Kidney disease (yes) & $4(2.36 \%)$ & $7(2.16 \%)$ & 0.8 \\
\hline \multicolumn{4}{|c|}{ Having close contact with defined COVID-19 Patient(s) within 6 months ago } \\
\hline Yes & $131(77.51 \%)$ & $204(63.15 \%)$ & \multirow{2}{*}{0.002} \\
\hline No & $38(22.48 \%)$ & $119(36.84 \%)$ & \\
\hline \multicolumn{4}{|c|}{ Having COVID-19 symptom(s) within six months ago } \\
\hline Cough & $48(28.40 \%)$ & $65(20.12 \%)$ & 0.001 \\
\hline Fever & $26(15.38 \%)$ & $44(13.62 \%)$ & 0.54 \\
\hline Chills & $33(19.52 \%)$ & $38(11.76 \%)$ & 0.01 \\
\hline Sore throat & $53(31.36 \%)$ & $83(25.69 \%)$ & 0.1 \\
\hline Headache & $68(40.23 \%)$ & $95(29.41 \%)$ & 0.01 \\
\hline Shortness of breath & $31(18.34 \%)$ & $39(12.07 \%)$ & 0.04 \\
\hline Diarrhea & $25(14.79 \%)$ & $41(12.69 \%)$ & 0.46 \\
\hline Chest Pain & $31(18.34 \%)$ & $40(12.38 \%)$ & 0.06 \\
\hline Decrease smell and taste & $17(10.05 \%)$ & $23(7.12 \%)$ & 0.23 \\
\hline Conjunctivitis & $21(12.42 \%)$ & $36(11.14 \%)$ & 0.62 \\
\hline Weakness & $63(37.27 \%)$ & $90(27.86 \%)$ & 0.02 \\
\hline History of COVID-19 positive (yes) & $120(71.00 \%)$ & $242(74.92 \%)$ & 0.001 \\
\hline \multicolumn{4}{|l|}{ ABO blood group } \\
\hline A & $39(23.07 \%)$ & $72(22.29 \%)$ & \multirow{4}{*}{0.18} \\
\hline $\mathrm{B}$ & $44(26.03 \%)$ & $63(19.50 \%)$ & \\
\hline $\mathrm{AB}$ & $16(9.46 \%)$ & $29(8.97 \%)$ & \\
\hline $\mathrm{O}$ & $55(32.54 \%)$ & $124(38.39 \%)$ & \\
\hline
\end{tabular}

were frontline staff. 35 (54.68\%) cases have COVID-19 symptoms and $42(65.62 \%)$ individuals had history of disease. No related was seen between blood group and immunoglobulin positivity. More details are provided in Table 3. Seropositivity prevalence in low risk and highrisk populations were estimated 8.0 (95\% CI 1.0-16.8) and 5.2 (95\% CI 1.2-9.8) respectively.

\section{Discussion}

This study was conducted to assess the state of COVID-19 epidemic in Shiraz, Iran. All what appears the end of 3rd waive before vaccination program. The preliminary results of this study provide an important benchmark to assess the state of the COVID-19 epidemic. Based on currents results, seropositivity prevalence in non-front line workers was higher than frontline workers. Various studies have assessed the seroprevalence of SARSCOV-2 in health care staff. According to few reports the seroprevalence varies widely between 33\% in New York, USA; $7.4 \%$ in Milan, Italy; $2 \%$ in Wuhan, China; and 1.2\% 
Table 2. Univariable Analysis of Associated Factors with Immunoglobulin G Against COVID-19

\begin{tabular}{|c|c|c|c|}
\hline & IgG Positive $(n=64)$ & IgG Negative $(n=428)$ & P Value \\
\hline Front-line physicians and nurses & $27(42.18 \%)$ & $142(33.17 \%)$ & \\
\hline Non-front-line health care workers & $37(57.81 \%)$ & $286(66.82 \%)$ & 0.13 \\
\hline \multicolumn{4}{|l|}{ Gender } \\
\hline Male & $34(53.12 \%)$ & $166(38.78 \%)$ & \multirow{2}{*}{0.02} \\
\hline Female & $30(46.87 \%)$ & $262(61.21 \%)$ & \\
\hline Age & $39.32 \pm 8.80$ & $42.47 \pm 68.88$ & 0.12 \\
\hline \multicolumn{4}{|l|}{ Underlying disease } \\
\hline Yes & $49(76.56 \%)$ & $312(72.89 \%)$ & \multirow[t]{2}{*}{0.53} \\
\hline No & $15(23.43 \%)$ & $116(21.10 \%)$ & \\
\hline \multicolumn{4}{|l|}{ Having symptoms } \\
\hline Yes & $35(54.68 \%)$ & $90(21.02 \%)$ & \multirow[t]{2}{*}{0.001} \\
\hline No & $29(45.31 \%)$ & $338(78.97 \%)$ & \\
\hline \multicolumn{4}{|l|}{ History of COVID-19 positive } \\
\hline Yes & $42(65.62 \%)$ & $103(24.06 \%)$ & \multirow{2}{*}{0.002} \\
\hline No & $22(34.37 \%)$ & $325(75.93 \%)$ & \\
\hline \multicolumn{4}{|l|}{$\mathrm{ABO}$ blood group } \\
\hline A & $20(31.25 \%)$ & $141(32.94 \%)$ & \multirow{4}{*}{0.53} \\
\hline B & $14(21.87 \%)$ & $93(21.72 \%)$ & \\
\hline$A B$ & $3(4.68 \%)$ & $42(9.81 \%)$ & \\
\hline $\mathrm{O}$ & $27(42.18 \%)$ & $152(35.51 \%)$ & \\
\hline
\end{tabular}

Table 3. Univariable Analysis of Associated Factors With Immunoglobulin M Against COVID-19

\begin{tabular}{|c|c|c|c|}
\hline Variables & IgM Positive $(n=37)$ & IgM Negative $(n=455)$ & P Value \\
\hline Front-line physicians and nurses & $14(37.83 \%)$ & $155(34.06 \%)$ & \\
\hline Non-front-line health care workers & $23(62.16 \%)$ & $300(65.93 \%)$ & 0.64 \\
\hline \multicolumn{4}{|l|}{ Sex } \\
\hline Male & $19(51.35 \%)$ & $273(60 \%)$ & \multirow{2}{*}{0.303} \\
\hline Female & $18(48.64 \%)$ & $182(40 \%)$ & \\
\hline Age & $42.53 \pm 67.08$ & $36.55 \pm 6.70$ & \\
\hline \multicolumn{4}{|l|}{ Underlying disease } \\
\hline Yes & $28(75.67 \%)$ & $333(73.18 \%)$ & \multirow{2}{*}{0.74} \\
\hline No & $9(24.32 \%)$ & $122(26.81 \%)$ & \\
\hline \multicolumn{4}{|l|}{ Having symptoms } \\
\hline Yes & $24(64.86 \%)$ & $343(75.38 \%)$ & \multirow{2}{*}{0.15} \\
\hline No & $13(35.13 \%)$ & $112(24.61 \%)$ & \\
\hline \multicolumn{4}{|l|}{ History of COVID-19 positive } \\
\hline Yes & $19(51.35 \%)$ & $98(21.53 \%)$ & \multirow{2}{*}{0.34} \\
\hline No & $18(48.64 \%)$ & $357(78.46 \%)$ & \\
\hline \multicolumn{4}{|l|}{ ABO blood group } \\
\hline A & $9(24.32 \%)$ & $150(32.96 \%)$ & \multirow{4}{*}{0.23} \\
\hline B & $12(32.43 \%)$ & $95(20.87 \%)$ & \\
\hline $\mathrm{AB}$ & $2(5.40 \%)$ & $45(9.89 \%)$ & \\
\hline $\mathrm{O}$ & $14(37.83 \%)$ & $165(36.26 \%)$ & \\
\hline
\end{tabular}

114 International Journal of Basic Science in Medicine. Volume 6, Issue 3, 2021 
in Essen city in Germany. ${ }^{8-11}$ These differences may be due to disease burden in countries and various methodologies. For instance; one of the survey was conducted two weeks after the first waive, this is while another study was performed two weeks before the first waive. Our study was conducted at the end of 3 rd waive. In fact, there are several factors which may effect on antibody prevalence. For instance; intensity or duration of exposure, the phase of epidemic, immunity of participants are important end effective factor on sero-prevalence.

Based on the current results, it was found; most of cases had history of COVID-19 disease within six months before this survey. In other study, it was found IgG antibodies were positive in $90 \%$ of cases who confirmed with COVID-19 two weeks after the onset of symptoms. ${ }^{12}$

A major finding in the current study is that high risk population which means frontline healthcare workers have lower seropositivity. This result was similar to Schmidt's study. None of the nurses and physicians turned out to be seropositive. Although they had long and close contact with positive COVID-19 patients. ${ }^{13}$ Furthermore, based on WHO guideline; it is expected that frontline public workers are expose to SARS-COV-2 more than others. In a conducted study in the United Kingdom; it was found the risk of getting COVID-19 disease was more than seven fold higher among health care staff. ${ }^{14}$ on the other hand, according to similar survey in Guilan province; type of occupation is not an independent risk factor for getting SARS-COV-2 infection. ${ }^{15}$ This finding raises the probability that seropositive staff might have infected outside the hospital.

One of the important points in the current study was non-significant association between blood groups and seropositivity, which is in contrast with another study that shown the sero-status and blood type is associated. It was declared that the proportion of seropositivity was significantly lower in blood type O. ${ }^{16}$ In Dai's report, it was revealed an association between blood type and susceptibility to infection. ${ }^{17}$ But another study published similar results with current study and mentioned that blood type is not a risk factor for getting SARS-COV-2 infection. ${ }^{17}$

Our study has some limitations that should be noted: First, it was better to select some cases and compare the results with general population. Second, it was the first time that the Pishtaz Teb SARS-CoV-2 ELISA kits were used for evaluation and it needs to perform more tests under monitored condition.

\section{Conclusion}

In conclusion, this study was an initial phase of an assay which aims to evaluate the sero-immunity of HCWs in central COVID-19 hospitals before the vaccination program and it is suggested to compare after the vaccination. The findings of this study imply that seropositivity was lower in non-frontline health care staff. Furthermore, there was no association between blood type and seropositivity. Therefore, the knowledge of antibody status may help to overcome fears in clinical staff, and also more attention is required for interpreting the levels of anti-SARS-CoV-2 antibodies to make a clinical diagnosis of COVID-19, since the time of expose and time of test are two important points which should be considered in the issue.

\section{Ethical Approval}

This study was approved by the ethical committee of Shiraz University of Medical Sciences (No. IR.SUMS.REC.1399.1192)

\section{Competing Interests}

The authors have no conflict of interest to declare.

Funding

The work has been covered with personal budget.

\section{Acknowledgments}

The authors would like to extend their gratitude to Nemazee hospital staff, affiliated with Shiraz University of Medical Sciences for their collaboration.

\section{References}

1. Ralph R, Lew J, Zeng T, et al. 2019-nCoV (Wuhan virus), a novel coronavirus: human-to-human transmission, travelrelated cases, and vaccine readiness. J Infect Dev Ctries. 2020;14(1):3-17. doi:10.3855/jidc.12425

2. $\mathrm{Xu} \mathrm{Z}$, Shi L, Wang Y, et al. Pathological findings of COVID-19 associated with acute respiratory distress syndrome. Lancet Respir Med. 2020;8(4):420-422. doi:10.1016/s22132600(20)30076-x

3. World Health Organization (WHO). Coronavirus Disease 2019 (COVID-19): Situation Report, 94. WHO; 2020.

4. Arshad Ali S, Baloch M, Ahmed N, Arshad Ali A, Iqbal A. The outbreak of coronavirus disease 2019 (COVID-19)an emerging global health threat. J Infect Public Health. 2020;13(4):644-646. doi:10.1016/j.jiph.2020.02.033

5. Donnelly CA, Ghani AC, Leung GM, et al. Epidemiological determinants of spread of causal agent of severeacuterespiratory syndrome in Hong Kong. Lancet. 2003;361(9371):17611766. doi:10.1016/s0140-6736(03)13410-1

6. Abbasi J. The promise and peril of antibody testing for COVID-19. JAMA. 2020;323(19):1881-1883. doi:10.1001/ jama.2020.6170

7. Shah J, Liu S, Potula $\mathrm{HH}$, et al. IgG and IgM antibody formation to spike and nucleocapsid proteins in COVID-19 characterized by multiplex immunoblot assays. BMC Infect Dis. 2021;21(1):325. doi:10.1186/s12879-021-06031-9

8. Rosenberg ES, Tesoriero JM, Rosenthal EM, et al. Cumulative incidence and diagnosis of SARS-CoV-2 infection in New York. Ann Epidemiol. 2020;48:23-29.e24. doi:10.1016/j. annepidem.2020.06.004

9. Bendavid E, Mulaney B, Sood N, et al. COVID-19 antibody seroprevalence in Santa Clara county, California. Int J Epidemiol. 2021;50(2):410-419. doi:10.1093/ije/dyab010

10. Dimcheff DE, Schildhouse RJ, Hausman MS, et al. Seroprevalence of severe acute respiratory syndrome coronavirus-2 (SARS-CoV-2) infection among Veterans Affairs healthcare system employees suggests higher risk of infection when exposed to SARS-CoV-2 outside the work environment. Infect Control Hosp Epidemiol. 2021;42(4):392-398. doi:10.1017/ice.2020.1220 
11. Sotgiu G, Barassi A, Miozzo $M$, et al. SARS-CoV-2 specific serological pattern in healthcare workers of an Italian COVID-19 forefront hospital. BMC Pulm Med. 2020;20(1):203. doi:10.1186/s12890-020-01237-0

12. Zhao J, Yuan Q, Wang $\mathrm{H}$, et al. Antibody responses to SARSCoV-2 in patients with novel coronavirus disease 2019. Clin Infect Dis. 2020;71(16):2027-2034. doi:10.1093/cid/ciaa344

13. Schmidt SB, Grüter L, Boltzmann M, Rollnik JD. Prevalence of serum IgG antibodies against SARS-CoV-2 among clinic staff. PLoS One. 2020;15(6):e0235417. doi:10.1371/journal. pone.0235417

14. Mutambudzi M, Niedwiedz C, Macdonald EB, et al. Occupation and risk of severe COVID-19: prospective cohort study of 120075 UK Biobank participants. Occup Environ Med. 2020;78(5):307-314. doi:10.1136/oemed-2020-106731

15. Shakiba M, Nazemipour M, Salari A, et al. Seroprevalence of SARS-CoV-2 in Guilan province, Iran, April 2020. Emerg Infect Dis. 2021;27(2):636-638. doi:10.3201/eid2702.201960

16. Gallian P, Pastorino B, Morel P, Chiaroni J, Ninove L, de Lamballerie $X$. Lower prevalence of antibodies neutralizing SARS-CoV-2 in group O French blood donors. Antiviral Res. 2020;181:104880. doi:10.1016/j.antiviral.2020.104880

17. Dai X. ABO blood group predisposes to COVID-19 severity and cardiovascular diseases. Eur J Prev Cardiol. 2020;27(13):1436-1437. doi:10.1177/2047487320922370 